\title{
Case Report \\ Acute gastric dilatation causing fatal outcome in a young female with eating disorder -a case report-
}

\author{
Seung-Mok Youm ${ }^{1,2}$, Ji Young Kim ${ }^{1,3}$, and Jeong Rim Lee ${ }^{1,3}$ \\ ${ }^{1}$ Department of Anesthesiology and Pain Medicine, ${ }^{2}$ Severance Hospital, ${ }^{3}$ Anesthesia and Pain Research Institute, \\ Yonsei University College of Medicine, Seoul, Korea
}

A 21-year-old female with a history of bulimia nervosa came to the emergency room due to severe abdominal pain after excessive eating five hours previously. On arrival at the emergency room, extreme abdominal distension was detected and the patient's legs changed color. Computed tomography suggested severe gastric dilatation, so abdominal compartment syndrome was suspected and an emergent laparotomy was supposed to be conducted. Though anesthesia was induced without event, abrupt hemodynamic collapse developed just after the operation started. In spite of active resuscitation for $29 \mathrm{~min}$, the patient did not recover and expired. As the incidence of eating disorders is increasing, anesthesiologists should keep in mind the possibility of abdominal compartment syndrome in patients with a recent history of binge eating, and prepare optimal anesthetic and resuscitation remedies against sudden deteriorations of a patient's condition.

Key Words: Abdominal compartment syndrome, Acute gastric distension, Eating disorders.

Anorexia-bulimia is a common psychiatric affliction in the adolescent female populations [1]. An increasing occurrence of eating disorders in non-western countries has been associated with cultural transition and globalization, including modernization, urbanization, and media-exposure promoting the western beauty ideal. Despite the lower prevalence of obesity in Korea compared with Western countries so far, the incidence of eating

Corresponding author: Jeong Rim Lee, M.D., Ph.D.

Department of Anesthesiology and Pain Medicine, Anesthesia and Pain Research Institute, Yonsei University College of Medicine, 50,

Yonsei-ro, Seodaemun-gu, Seoul 120-752, Korea

Tel: 82-2-2227-3840, Fax: 82-2-2227-7897

E-mail: manya@yuhs.ac

Received: May 26, 2014.

Revised: 1st, July 3, 2014; 2nd, July 8, 2014.

Accepted: July 29, 2014.

Korean J Anesthesiol 2015 April 68(2): 188-192

http://dx.doi.org/10.4097/kjae.2015.68.2.188 disorders in Korean adolescents has been increasing, especially female teens [2].

Though infrequent, errant eating behavior results in acute gastric distension (AGD), which can induce abdominal compartment syndrome (ACS) [3]. As acute gastric dilation progresses, intra-abdominal pressure (IAP) is increased, and the viability of multiple organs is threatened when the effective circulatory volume is decreased. When severe, it may end in hemodynamic instability, shock, or even death. Therefore, eating disorders may have a higher incidence of fatal outcomes than expected, because of episodes of acute gastric dilatation followed by ACS.

We experienced the intra-operative death of the young female patient with severe gastric distension, who had suffered from an eating disorder. Though eating disorders have become serious social and medical issues in our country, most clinicians may not perceive eating disorders as the cause of life-threatening situations. Therefore, we would like to introduce and discuss this case so that anesthesiologists have knowledge of rare but possible serious complications and are better able to cope with that.

(c) This is an open-access article distributed under the terms of the Creative Commons Attribution Non-Commercial License (http://creativecommons.org/ licenses/by-nc/3.0/), which permits unrestricted non-commercial use, distribution, and reproduction in any medium, provided the original work is properly cited. 


\section{Case Report}

A 21-year-old female patient $(162 \mathrm{~cm}, 48.4 \mathrm{~kg})$ visited the emergency room with abdominal pain after eating a heavy meal five hours previously. She had been treated at a local psychiatric clinic for anorexia nervosa and bulimia nervosa, and she had a relatively low body mass index of $18.44 \mathrm{~kg} / \mathrm{m}^{2}$.

While visiting the emergency room, her abdomen looked markedly distended. She suffered from nausea and retching but was unable to vomit. Her vital signs upon arrival at the emergency room were as follows: Blood pressure $128 / 83 \mathrm{mmHg}$, pulse 78 beats/min, respiratory rate 18 breaths/min, and a body temperature of $36.8^{\circ} \mathrm{C}$. Chest AP and simple abdomen flat upright view revealed that the stomach was full of food material with air-fluid level. Computed tomography showed a massive gastric overdistension with pneumoperitoneum (Fig. 1). A Foley catheter was inserted into the bladder cavity, and the amount of urine there was nearly zero (Fig. 2). Levin tube insertion revealed that the material in her stomach was incompletely digested and that drainage was not effective. The initial complete blood cell count was within normal ranges, including a hemoglobin level of $12 \mathrm{~g} / \mathrm{dl}$. Blood chemistry had no unusual characteristics except doubly elevated levels of amylase and lipase.

While going through the various examinations, the emergency specialist noticed that her both lower extremities were turn to be cyanosis. The pulsation of both lower extremities could not be checked. Furthermore, her heart rate was changed to a tachycardic rate of 135-143 beats/min two hours after it was first checked. Therefore, the surgeon decided to perform an emergency exploratory laparotomy in the operating room.

As the patient entered the operating room, she displayed a slightly drowsy mental disorder with fast and shallow respiration. Her approximate breathing rate was more than 30 breaths/ $\min$.

We applied invasive arterial pressure monitoring with a $20 \mathrm{G}$ angiocatheter on her right radial artery and inserted a 7 French central line catheter on her left internal jugular vein subsequently, prior to induction. Just as before the induction, her blood
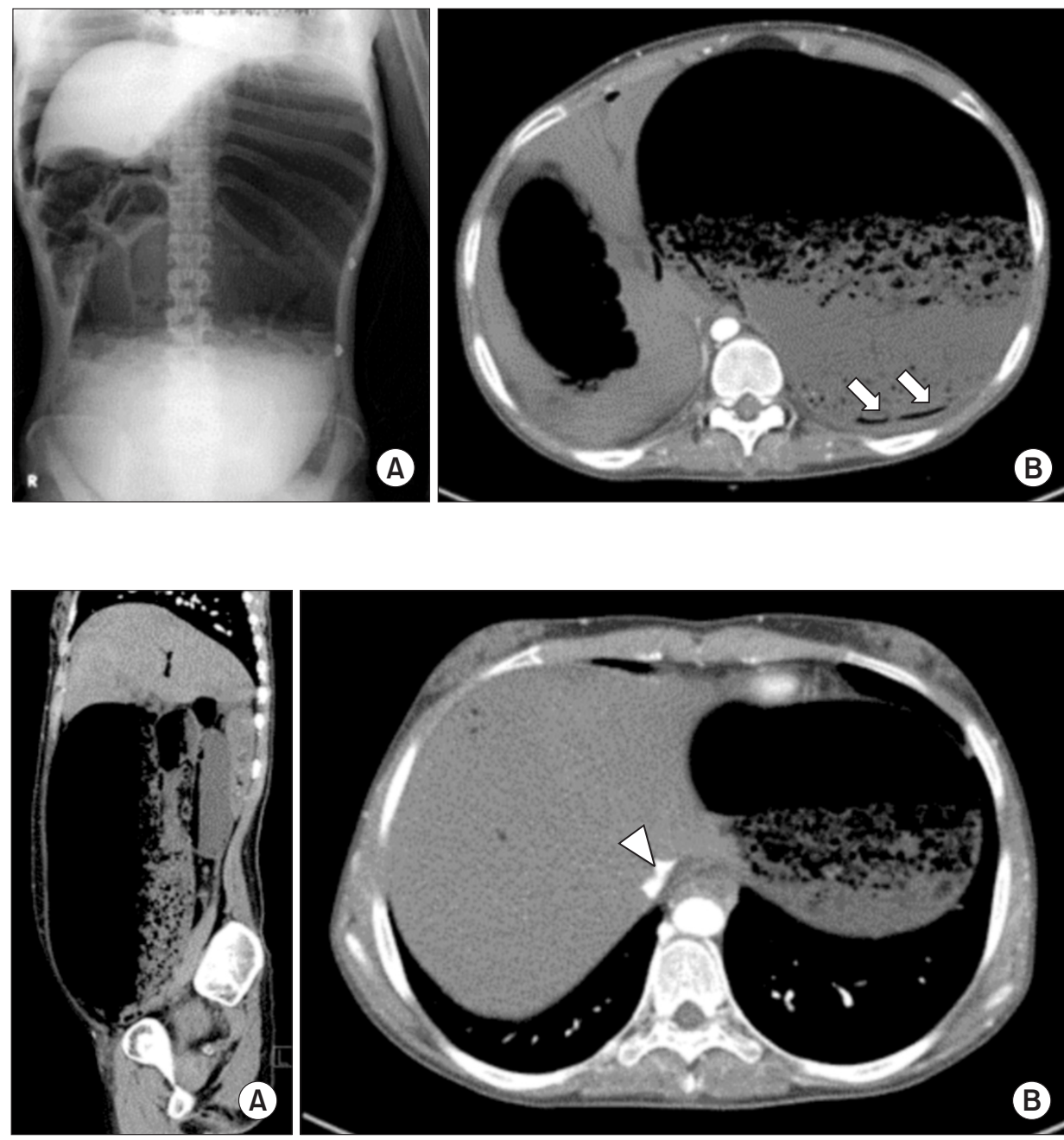

B
Fig. 1. (A) Simple upright abdominal radiography shows that markedly distended stomach with food materials occupying almost entire abdominal cavity. Note that there is moderate amount of pneumoperitoneum, suggesting gastric perforation. (B) Markedly distended stomach with food material. Note that pneumatosis along the gastric wall, suggesting infarction (white arrows). 
Table 1. Arterial Blood Gas Analysis through the Laparotomy

\begin{tabular}{lcccccc}
\hline & $\mathrm{pH}$ & $\begin{array}{c}\mathrm{pCO}_{2} \\
(\mathrm{mmHg})\end{array}$ & $\begin{array}{c}\mathrm{HCO}_{3} \\
(\mathrm{mmHg})\end{array}$ & $\begin{array}{c}\mathrm{BE}-\mathrm{ECF} \\
(\mathrm{mmol} / \mathrm{L})\end{array}$ & $\begin{array}{c}\mathrm{BE}-\mathrm{B} \\
(\mathrm{mmol} / \mathrm{L})\end{array}$ & $\begin{array}{c}\mathrm{Hb} \\
(\mathrm{g} / \mathrm{dl})\end{array}$ \\
\hline $\begin{array}{l}\text { Operation starts } \\
\begin{array}{l}\text { 12 minutes after the initiation of operation } \\
\text { (cardiac compressions start) }\end{array}\end{array}$ & 7.297 & 27.0 & 13.3 & -13.4 & -11.4 & 6.4 \\
20 minutes after the initiation of cardiac compressions & 7.063 & 47.6 & 10.9 & -21.3 & -18.6 & 6.1 \\
\end{tabular}

BE-ECF: base excess in extracellular fluid compartment, BE-B: Base excess in blood, Hb: Hemoglobin.

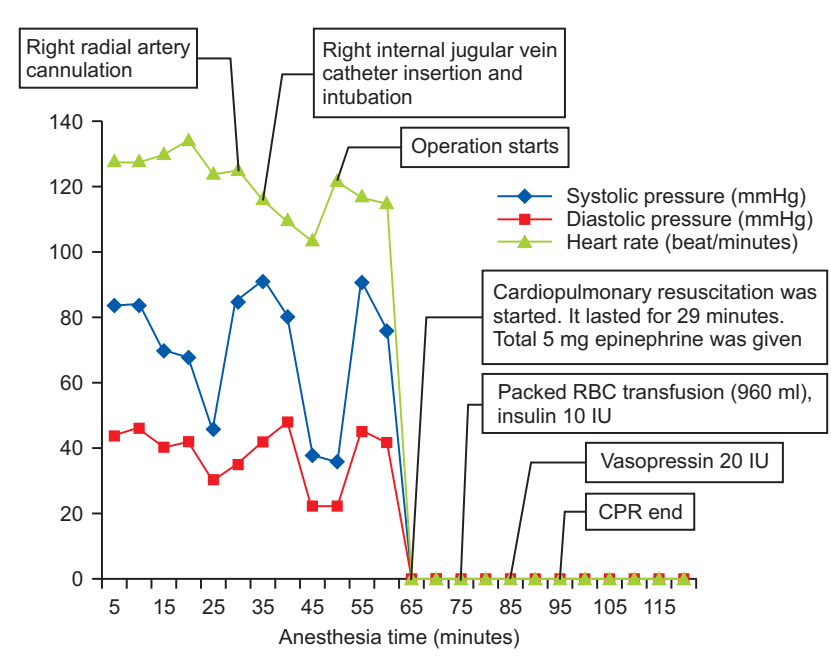

Fig. 3. The chart shows that the systolic pressure, diastolic pressure, pulse rate and the events of perioperative period. CPR: cardiac pulmonary resuscitation.

pressure dropped to $80 / 48 \mathrm{mmHg}$, her heart rate was 110 beats/ min, and her mental status turned to a stupor. We tried to oxygenate the patient with a mask fitting and insufflations of $100 \%$ oxygen for several minutes. Anesthetic induction was conducted with midazolam $3 \mathrm{mg}$ and then rocuronium $50 \mathrm{mg}$ IV bolus, and $1 \mathrm{~min}$ after endotracheal intubation was performed without prior positive pressure ventilation with the facial mask. Just after intubation, the patient's vital signs were tolerable, with a blood pressure of $91 / 42 \mathrm{mmHg}$ and a heart rate of 116 beats/min. For anesthetic maintenance, sevoflurane inhalation with remifentanil continuous infusion was started. A few minutes after induction, the first arterial blood sample for blood gas analysis was drawn, and the results indicated both mixed metabolic acidosis and respiratory alkalosis (Table 1). Hyperkalemia was also detected at $7.4 \mathrm{mmol} / \mathrm{L}$, so the anesthesiologist ordered that a nurse get calcium, sodium bicarbonate, and insulin. At that time, the anesthesiologists noticed that the electrocardiogram readings seemed abnormal. Meanwhile, just after the abdominal cavity was opened and the gastric contents were sucked out through a small gastric incision, her blood pressure abruptly dropped to $36 / 22 \mathrm{mmHg}$ (Fig. 3), and the electrocardiogram showed

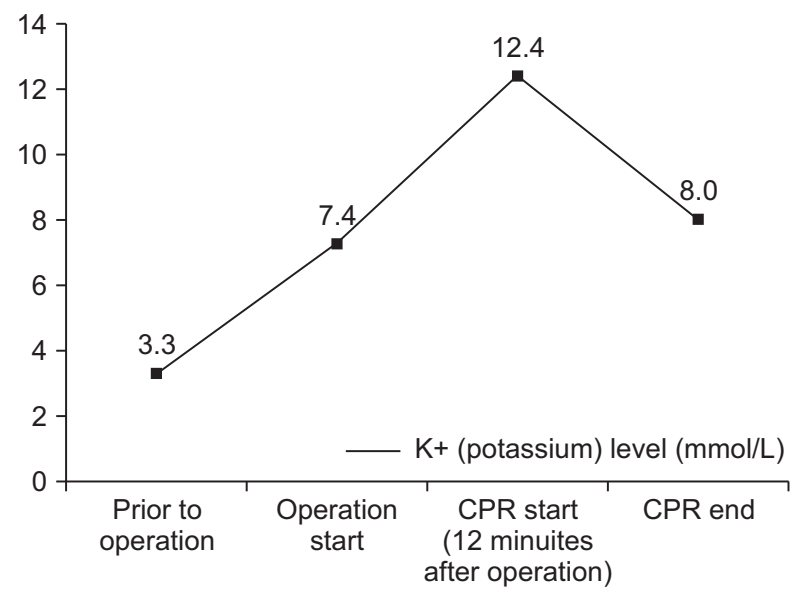

Fig. 4. The potassium levels during the laparotomy. It shows that the potassium level was extremely increased just after the initiation of the surgery.

bizarre and wide QRS. Immediate treatment was conducted, which included the rapid infusion of $800 \mathrm{ml}$ of crystalloid and $400 \mathrm{ml}$ of colloid fluid, the start of a continuous infusion of norepinephrine at $0.3 \mu \mathrm{g} / \mathrm{kg} / \mathrm{min}$, the bolus administration of $300 \mathrm{mg}$ of calcium, and $20 \mathrm{mEq}$ of sodium bicarbonate. However, her tremendously decreased blood pressure could not be restored and her cardiac rhythm became asystolic. It only took 12 minutes from the initiation of the exploratory laparotomy to asystole. We started cardiac compressions immediately, and during the cardiac compression for resuscitation, five $1 \mathrm{mg}$ doses of epinephrine bolus injection were given at a rate of one every few minutes. A bolus of $20 \mathrm{IU}$ of vasopressin was also injected after the administration of the fourth dose of epinephrine. Insulin 10 IU was also administered, and both $300 \mathrm{mg}$ of calcium and 300 $\mathrm{mEq}$ of sodium bicarbonate were injected approximately every $5 \mathrm{~min}$, and so a total of $1,200 \mathrm{mg}$ of calcium chloride and 1,200 $\mathrm{mEq}$ of sodium bicarbonate were administered to correct the hyperkalemia. Even though the procedure made for less than 50 $\mathrm{ml}$ of blood loss, four units of packed RBC were transfused for the decreasing tendency of hemoglobin down to $4.9 \mathrm{~g} / \mathrm{dl}$. Additional fluids as well as furosemide $40 \mathrm{mg}$ IV bolus were given to correct the anuria. Consequently, as much as a total of 1,200 ml of crystalloid and $600 \mathrm{ml}$ of colloid, which were loaded with 960 
$\mathrm{ml}$ packed $\mathrm{RBC}$ were given during the cardiac resuscitation. The results of the arterial blood gas analysis throughout the anesthesia and resuscitation are presented in Table 1, and the change of potassium is presented in Fig. 4. All efforts for resuscitation including cardiac compressions were maintained for 29 minutes. Despite these exertion, the asystole state persisted, her blood pressure was not checked and no cardiac rhythm was restored, so the surgeon in charge pronounced her dead. The operating note illustrated that not only the stomach but also the small bowel and colon showed color change, suggesting necrosis. There was no description of the other solid abdominal organs.

\section{Discussion}

It is generally thought that eating disorders are just psychological problems and far from life-threatening conditions. However, though rare, binge eating after fasting is able to induce severe gastric distension, which is a real, fatal condition requiring prompt emergency medical and surgical intervention. Frequent or prolonged fasting gradually makes the gastric wall thin, decreases gastric motility, and delays gastric emptying [4], so the stomach cannot endure a large amount of food being eaten abruptly. Ingested food fails to be digested and passed to the intestine, and this AGD results in an increase in the intraabdominal pressure. Finally, the patient suffers from an ischemic injury of various intra-abdominal organs, depending on the degree of intra-abdominal pressure and the compression of the inferior vena cava or aorta can induce a loss of circulatory volume depending on the degree of IAP.

According to classification by the World Society of the Abdominal Compartment Syndrome, ACS may be defined as a sustained IAP of $>20 \mathrm{mmHg}$ with the presence of an attributable organ failure [5], and the mortality in grade IV IAP ( $>25$ $\mathrm{mmHg}$ ) is known to be $71-85 \%$. Some cases may end in sudden death before coming to the hospital [6]. In the present case, the emergency specialist tried to check for intra-abdominal pressure through intra-vesicular pressure, but failed due to anuria [7]. Acute renal failure is thought to have already occurred; therefore, IAP would be more than $20 \mathrm{mmHg}$. In a real clinical setting, prior measurement of the IAP and treatment according to IAP is unlikely to be practical. A proper treatment strategy has to be performed promptly if AGD with ACS is suspected.

In a few cases, the symptom was relieved after stomach decompression through a nasogastric tube or gastroendoscopy [8]. However, in our case, nasogastric decompression was impossible because the food material that was not digested was too thick. In addition, the insertion of a nasogastric tube or gastric lavage tube may be impossible due to the occlusion of the gastroesophageal junction by the distended fundus, which angulates the esophagus against the right crus of the diaphragm [9]. For the same reason, a patient feels that it is too difficult to vomit, despite nausea or emesis. The present patient also failed to vomit.

Surgical abdominal decompression has long been the standard treatment for patients who develop ACS. Although the decompression of the stomach through a nasogastric tube succeeded, the wall of stomach had already been weakened, so it underwent ischemic change, and finally necrosis and perforation. Therefore, the injured stomach should be resected in most cases after all [8]. However, even though operation was successfully completed, most reported fatal outcomes occurred in the subacute stage. Kim et al. [9] reported a mortality case regarding abdominal compartment syndrome that was caused by a bulimic attack. According to his report, CT findings before surgery showed the collapse of major vessels including the aorta and the infarction of kidney, spleen, pancreas, liver, small bowel, and both transverse and descending colon. Despite successful decompression, cardiovascular compromise developed, and there was oozing bleeding from the gastric cavity and peritoneal cavity, which were unable to be controlled. The authors assumed that the cause of death was septic shock caused by the ischemiareperfusion injury. Gyurkovics et al. [10] reported other mortality case of DIC related with reperfusion injury. In this case, the time taken for the diagnosis and treatment was more than six hours, and she died more than 24 hours after surgery, due to hypovolemia, which was related with sepsis [10]. Another fatal case reported by Turan et al. [11] concluded that the cause of death was bacteremia and shock, caused by a massively gangrenous stomach. As such, in the published cases, subacute septic shock is presumed to be the most common cause of death. When compared with the other lethal cases, our present case appears different because the cardiovascular collapse following asystole was abruptly developed soon after decompression. Of course, the possibility of reperfusion after severe ischemia has been identified [12]. The release of intra-abdominal pressure induces a massive bloodstream inflow of under-oxidized decay products, and its extent may be related to the pressure $[9,12]$. Therefore, the main leading cause of death is thought to be the sustained release of an unexpectedly large amount of potassium to the circulation from ischemic tissue. Moreover, her body could not compensate for her hyperkalemia; acute kidney injury might have already developed since her urine output was nearly zero for a couple of hours. We failed to predict lethal hyperkalemia in advance for several reasons: Her electrolyte analysis findings in the emergency room were almost normal, and there had not been any reported cases of AGD with fatal hyperkalemia. As soon as we noticed this problem through blood gas analysis, which was checked at the start of the operation, we prepared several drugs including calcium, bicarbonate, and insulin. However, before we started treatment, the surgeon conducted a full decompression. Finally, reperfusion followed by intractable hy- 
perkalemia combined with acidosis would develop. At that time, the empirical use of calcium, sodium bicarbonate, and insulin was not enough to reverse the situation. In summation, the combination of an unexpected amount of potassium released just after decompression, combined with the acidosis, and accompanied by the acute kidney injury is thought to have combined to lead to the failure of the treatment of hyperkalemia. We should have requested that the surgeon delay any decompression procedure until the hyperkalemia was fully corrected and further treatment modality would have been available. The final treatment method for hyperkalemia would be renal replacement therapy (RRT) [13]. Because it is possible to prepare and apply RRT rapidly in such a pressing situation, this condition should have been anticipated, and treatment should have been prepared beforehand.

Survival or death would be determined according to the time taken diagnosis, intervention and the extent of ischemic change/ ischemia-reperfusion. One patients with a more fast administration (1 hour after binge eating) survived after an intervention of gastric decompression and surgery of a partial gastrectomy [4]. The other patient administered under shock with 8-10 hours of symptoms also survived; they did not suffer from symptoms of leg ischemia, were able to vomit, and a nasogastric tube was successfully inserted [14]. However, there has been no consensus of when surgical decompression has to intervene, and what examination should be taken before the decision of treatment. According to a few case reports, surgery was performed immediately afterwards, just when AGD with ACS was suspected, while in the other cases, the computed tomography was checked in advance. One more thing that should be of concern is that the stable vital signs of a patient in the emergency room may delay the decision of surgeons to perform a laparotomy, as happened in our case.

Abdominal compartment syndrome has already been identified as a serious condition for clinicians; we also have to know that this syndrome can be the result of binge eating or start abruptly for patients with psychological eating disorders. In a real situation, a direct impression may be difficult, because many of these patients deny their history of psychological diseases $[4,15]$. Therefore, anesthesiologists have to keep in mind the possibility of a serious ACS in abnormal eating behavior, the necessity of a timely emergency laparotomy, and provide against every possible complication, including reperfusion syndrome with hyperkalemia and disseminated intravascular coagulation.

\section{References}

1. Smink FR, van Hoeken D, Hoek HW. Epidemiology of eating disorders: incidence, prevalence and mortality rates. Curr Psychiatry Rep 2012; 14: 406-14.

2. Lee HJ, Park S, Kim CI, Choi DW, Lee JS, Oh SM, et al. The association between disturbed eating behavior and socioeconomic status: the Online Korean Adolescent Panel Survey (OnKAPS). PLoS One 2013; 8: e57880.

3. Sugrue M, Hallal A, D’Amours S. Intra-abdominal pressure hypertension and the kidney. Abdominal Compartment Syndrome. Edited by Ivatury R, Cheatham M, Malbrain M, Sugrue M. Georgetown, Landes Bioscience. 2006, pp 119-28.

4. Tweed-Kent AM, Fagenholz PJ, Alam HB. Acute gastric dilatation in a patient with anorexia nervosa binge/purge subtype. J Emerg Trauma Shock 2010; 3: 403-5.

5. Malbrain ML, Cheatham ML, Kirkpatrick A, Sugrue M, Parr M, De Waele J, et al. Results from the International Conference of Experts on Intra-abdominal Hypertension and Abdominal Compartment Syndrome. I. Definitions. Intensive Care Med 2006; 32: 1722-32.

6. Watanabe S, Terazawa K, Asari M, Matsubara K, Shiono H, Shimizu K. An autopsy case of sudden death due to acute gastric dilatation without rupture. Forensic Sci Int 2008; 180: e6-10.

7. Balogh Z, De Waele JJ, Malbrain ML. Continuous intra-abdominal pressure monitoring. Acta Clin Belg Suppl 2007; (1): 26-32.

8. Van Eetvelde E, Verfaillie L, Van De Winkel N, Hubloue I. Acute gastric dilatation causing acute limb ischemia in an anorexia nervosa patient. J Emerg Med 2014; 46: e141-3.

9. Kim BS, Kwon JW, Kim MJ, Ahn SE, Park HC, Lee BH. Abdominal compartment syndrome caused by a bulimic attack in a bulimia nervosa patient. J Korean Surg Soc 2011; 81 Suppl 1: S1-5.

10. Gyurkovics E, Tihanyi B, Szijarto A, Kaliszky P, Temesi V, Hedvig SA, et al. Fatal outcome from extreme acute gastric dilation after an eating binge. Int J Eat Disord 2006; 39: 602-5.

11. Turan M, Sen M, Canbay E, Karadayi K, Yildiz E. Gastric necrosis and perforation caused by acute gastric dilatation: report of a case. Surg Today 2003; 33: 302-4.

12. Timerbulatov ShV, Timerbulatov VM, Fayazov RR, Sultanbaev AU, Gareev RN, Khalikov AA. Ischemia-reperfusion injury in intraabdominal hypertension in experiment and clinic. Dokl Biol Sci 2013; 451: 209-13.

13. Palevsky PM. Renal replacement therapy in acute kidney injury. Adv Chronic Kidney Dis 2013; 20: 76-84.

14. Dympep B, Singh CB, Shakeel M. Acute gastric dilatation with necrosis and perforation: case report of a 16 year old girl. Indian J Surg 2013; 75(Suppl 1): 180-1.

15. Paschold M, Gockel I, Oberholzer K, Lang H, Düber C. Pneumoabdomen with abdominal compartment and aortic collapse due to gastric bursting acute release by trocar insertion. Circulation 2013; 127: 417-8. 